\title{
Surgical Revascularization in Children with Sickle Cell Disease and Moyamoya Syndrome
}

\author{
Matthew Kruchten ${ }^{1}$, Sabrina Han ${ }^{1}$, Kelsey Hayward ${ }^{1}$, Joseph Piatt ${ }^{2}$, Corinna Schultz ${ }^{2}$, \\ Kelly Gassie ${ }^{3}$, Eliza Carroll ${ }^{4}$, Ricardo Hanel $^{5}$, and Philipp Aldana ${ }^{1}$ \\ ${ }^{1}$ University of Florida College of Medicine \\ ${ }^{2}$ AI DuPont Hospital for Children \\ ${ }^{3}$ Mayo Clinic Hospital Jacksonville \\ ${ }^{4}$ University of South Florida College of Public Health \\ ${ }^{5}$ Lyerly Neurosurgery
}

June 10, 2020

\begin{abstract}
Background: Pediatric patients with sickle cell disease (SCD) and moyamoya syndrome (MMS) are at high risk for recurrent cerebrovascular accidents (CVA) despite best conservative management with chronic blood transfusions (CBT). The role of surgical revascularization in this patient population is not clearly defined. We aim to compare the risk of stroke occurrence in patients undergoing conservative treatment to those additionally undergoing surgical revascularization with conservative treatment. Methods: The authors performed a retrospective cohort study of pediatric SCD-MMS patients seen in Jacksonville and Delaware between 2006 and 2018. Patient characteristics and outcomes, including stroke occurrence, were compared between those who underwent conservative treatment and those who underwent surgical revascularization. Results: A total 17 patients diagnosed with SCD-MMS were on CBT, of which $12(70.6 \%)$ were female. The average age at CBT initiation was 7.27 years. Prior to its initiation, 10 patients $(83 \%)$ in the surgical and $6(75 \%)$ in the conservative group experienced strokes. Twelve patients underwent surgical revascularization on 18 hemispheres (17 indirect, 1 direct) for severe vasculopathy or CVA - this included 2 patients who experienced a stroke while receiving CBT. The degree of vasculopathy was worse in the surgical group. The mean follow-up for conservative and surgical groups were 11.8 and 4.4 years, respectively. Three patients experienced strokes in the conservative group, while no strokes or major complications were observed in the surgical group. Conclusion: Surgical revascularization appears safe and may effectively reduce stroke rates in pediatric patients with SCD-MMS. Larger studies are needed to confirm these findings.
\end{abstract}

\section{Introduction:}

Stroke is among the most severe and common comorbidities experienced by children with sickle cell disease (SCD) with an estimated prevalence of $11 \%$ before the age of 20 years. ${ }^{14}$ Of these children with SCD who present with stroke, up to $43 \%$ are found to have moyamoya syndrome (MMS) on angiographic imaging. ${ }^{5}$ MMS is a cerebrovascular condition secondary to a pathologic condition, such as SCD, that is characterized by progressive stenosis of the arteries of the Circle of Willis and the development of a fragile network of small vessels that provide collateral blood flow. This network of capillary-sized blood vessels can fail to supply the necessary blood and oxygen to the brain, thus children with SCD with MMS are 5 times more likely to experience recurrent cerebrovascular events (CVEs - defined as strokes and transient ischemic attacks) compared to patients with SCD without MMS. ${ }^{5}$

Despite this increased risk of cerebral ischemia, treatment to prevent stroke that is directed at children with SCD-MMS has not been well studied. The current standard of care for stroke prevention is chronic 
transfusion therapy (CTT) to maintain a pre-transfusion sickle hemoglobin (HbS) level less than 30\%.5,15 This has demonstrated reduced rates of cerebrovascular events in pediatric patients with SCD, although the risk of stroke in children with SCD-MMS still remains significant. ${ }^{1,13}$ Additionally, CTT is associated with complications such as iron overload, compliance issues, and treatment discontinuation. ${ }^{3}$

Direct and indirect surgical revascularization procedures have been shown to be effective in reducing recurrent events in idiopathic MMS; however, their role and effectiveness in the treatment for the combination of SCD-MMS have not been well characterized. Small, single-center, retrospective studies in pediatric patients with SCD-MMS have suggested that surgical revascularization decreases the risk of stroke with minimal perioperative risk..$^{4,6,7,9,12,15-17}$ Despite promising results, these studies are limited by their small cohort size and lack of control groups. We sought to examine the outcomes in a larger cohort of children with SCD-MMS who underwent surgical revascularization in addition to CTT compared to those treated with CTT alone at two sickle cell treatment centers with active hematology and neurosurgery programs.

\section{Materials and Methods/Case Material:}

\section{Retrospective cohort study}

A retrospective cohort study examined pediatric patients (ages 0-18 years) on CTT at the sickle cell programs of the Nemours Alfred I. duPont Hospital for Children in Delaware and Nemours Specialty Care, University of Florida - Jacksonville and Wolfson Children's Hospital from 2006 to 2018. This study was approved by both institutional review boards with a waiver of informed consent. Patients diagnosed with SCD and MMS were included in the study. The diagnosis of MMS was determined by documented imaging findings on magnetic resonance angiography (MRA), computerized tomographic angiography or digital subtraction angiography. Diagnostic criteria of MMS on MRA includes characteristic angiographic findings of stenosis or occlusion of the terminal portion of the internal carotid artery and the proximal portions of the anterior cerebral and middle cerebral arteries along with abnormal collateral vessels at the base of the brain. ${ }^{10}$

Conservative and surgical treatment groups were defined retrospectively. The conservative treatment group consisted of patients treated with CTT and medical therapy, including aspirin and iron chelation. The surgical group consisted of patients treated with the same conservative methods with the addition of surgical cerebral revascularization (indirect or direct vascular bypass). All revascularizations were performed by the senior authors (P.A., R.H. and J.P.).

The follow-up period was defined as the interval between the first transfusion on CTT and the last followup appointment for patients in the conservative treatment group, or the interval between the first surgical treatment and the last follow-up appointment for patients in the surgical treatment group. The study endpoints included symptomatic stroke, stroke-related death, other cerebrovascular events [CVE - defined as asymptomatic stroke or transient ischemic attack (TIA)]. Major surgical complications within 90 days of surgery were reported.

Patient characteristics were compared in domains such as: age at first stroke and subsequent strokes, age at initiation of CTT, aspirin intake, and iron chelation therapy. Vasculopathy scores were graded by a pediatric neuroradiologist based on the vascular occlusion present on the patient's MRA imaging according to the SWiTCH trial. The trial standardized a grading scale from 0 to 6 to assess the location, extent and severity of vasculopathy, with increasing severity. ${ }^{11}$ A student T-test was used for continuous variables, a chi-square test was used for categorical variables, and the Wilcoxon rank-sum test for ordinal variables. Incidence of stroke recurrence was compared between the conservative treatment group and the surgical treatment group using a Kaplan-Meier survival curve and the Logrank test. Statistical significance was defined as $\mathrm{p}<0.05$.

\section{Revascularization procedure}

Our protocol for screening, medical management, and surgical management of patients with SCD and MMS has been described previously. ${ }^{2}$ Indications for surgery include recent stroke or transient ischemic attack 
(TIA) in the same distribution as cerebral arterial stenosis with the presence of moyamoya collaterals. Patients with remote or asymptomatic strokes generally undergo a physiologic cerebral flow study, such as single-photon emission computed tomography, followed by a similar study with acetazolamide. Following the administration of acetazolamide, if an area of hypoperfusion is found concordant with a stenosed cerebral artery, then revascularization surgery is generally recommended. In general, indirect revascularization procedures such as encephaloduro-arteriosynangiosis are performed; occasionally, direct superficial temporal artery to middle cerebral artery anastomosis has been performed, per surgeon's preference.

Results:

Patient population and characteristics

Seventeen patients with SCD-MMS were included in our study. Twelve patients $(70.6 \%)$ of the cohort were female. All patients were initially managed conservatively. Ten patients in the cohort received surgical revascularization at a later time (Figure 1). Eight patients $(80 \%)$ in the surgical group and six patients $(85.7 \%)$ in the conservative group presented with unilateral stroke before beginning conservative medical management. The average age at first stroke was 6.0 and 8.25 years old in the conservative and surgical treatment groups, respectively. Of the remaining patients, two (20\%) in the surgical group presented with TIA, and two $(28.6 \%)$ in the conservative group were asymptomatic but presented with an elevated peak flow velocity on Transcranial Doppler Ultrasound. When comparing the average vasculopathy scores of the left and right hemispheres, the mean and median score for the surgical group was 2.89 and 3, respectively. For the conservative group, the mean and median scores were generally lower, 1.58 and 1.5, respectively. The differences in pooled (left and right) mean vasculopathy scores between conservative and surgical groups were found to be statistically significant $(\mathrm{p}=0.04)$. Eight patients $(80 \%)$ in the surgical group and 5 patients $(71.4 \%)$ in the conservative group were on aspirin therapy. Nine patients $(90 \%)$ in the surgical group and 6 patients $(85.7 \%)$ in the conservative group were on iron chelation therapy (Figure 2). These differences between groups were not statistically significant. Further characterization of each patient is listed in Table 1.

Surgical revascularization and outcomes

The average follow-up period until last follow-up or stroke was 4.4 and 7.4 years for the surgical and conservative groups, respectively. In the surgical revascularization group, there were no strokes; one patient experienced a TIA. In the conservative treatment group, three patients experienced one stroke each (3 strokes per 100 patient years or $3 \%$ per year). In addition to CTT, two of the three conservative patients who experienced stroke were on aspirin therapy, and all three were on iron chelation therapy. Although the Kaplan-Meier survival analysis of stroke-free survival comparing the two groups showed a clear divergence between curves, it did not show a statistically significant difference $(\mathrm{p}=0.146)$ (Figure 3$)$.

\section{Discussion:}

This study represents, to our knowledge, the largest retrospective cohort of pediatric patients with SCD-MMS analyzing the effectiveness and safety of surgical revascularization compared to conservative therapy. After initiation of treatment, no strokes were observed in the ten patients who underwent surgical revascularization, while three of the seven patients who underwent conservative therapy experienced a stroke during the course of the average 7.4 year follow-up despite having less severe cerebral vasculopathy. No significant complications of surgery were observed.

Hankinson et al. examined 12 pediatric SCD-MMS patients who underwent surgical revascularization for an average of 46.8 months follow-up and found no strokes post-operatively and no major surgical complications. ${ }^{9}$ Only four of the patients were compliant with CTT protocol throughout the study; patients who received conservative treatment only were not included. Greissenhauer et al. examined a similar cohort of 14 pediatric SCD-MMS patients on CTT who underwent surgical revascularization. ${ }^{7}$ There was one observed stroke in the cohort and a reported 5 -fold reduction risk in stroke compared to pre-surgical risk. Our group recently published a systematic review of the literature available on surgical revascularization for children with sickle 
cell disease and moyamoya syndrome. We found an overall rate of ischemic stroke free survival of $94.3 \%$ with only $1.8 \%$ intraprocedural complications. ${ }^{2}$

A study comparing conservative and surgical treatment was published by Yang et al. who examined 15 pediatric SCD-MMS patients. ${ }^{17}$ Seven patients underwent surgical revascularization and eight patients underwent conservative therapy. The authors observed no strokes in the surgical revascularization group and four strokes in the conservative treatment group with an average follow-up of 11.6 years equating to a $2.3 \%$ risk of stroke per year. The results of our study showed very similar results to these studies. We observed a stroke risk of 3.0\% per year in SCD-MMS patients undergoing conservative therapy alone and no risk of stroke in patients who underwent surgical revascularization. When considering the differences in average vasculopathy scores and observing a statistically significant difference between the two groups, the evidence of more severe vasculopathy in the surgical group supports the protective nature of the surgery treatment in regards to stroke.

Although our study does not show a statistically significant difference between treatment options, there is a clear trend towards a clinically significant reduction of stroke risk associated with surgical revascularization. By combining our data with published data from the Yang et al. study ${ }^{17}$ and the Hall et al. study ${ }^{8}$ (the only studies with similar cohorts of patients and detailed outcomes), the pooled data showed a statistically significant difference $(\mathrm{p}=0.001)$ in the stroke rates between the conservative $(\mathrm{n}=30)$ and surgical $(\mathrm{n}=29)$ groups. Figure 4 shows the difference in survival curves with the surgical group showing superior results.

The major limitations of our study are the lack of statistical power due to small sample size and the lack of equivalent long-term follow-up in the surgical revascularization group. Furthermore, the two treatment groups are not likely to differ only in the surgical therapy due to the retrospective design of the study. A multi-center, retrospective study is currently underway to accrue a larger patient sample in order to better detect a difference in stroke outcome between conservative treatment and surgical revascularization.

\section{Conclusions:}

The results of our study indicate that surgical revascularization is safe and does not result in further strokes in pediatric patients with SCD-MMS. On the other hand, patients with the same diagnosis treated conservatively experienced strokes at a rate of $3 \%$ per year, however the difference between the two groups' stroke rates were not statistically significant. Larger multicenter prospective studies are warranted to further examine the utility and safety of surgical revascularization in this patient population.

Disclosures: The authors report no conflict of interest concerning the materials or methods used in this study or the findings specified in this paper.

Acknowledgments: We wish to acknowledge that this work has been supported in part by the Lucy B. Gooding Charitable Foundation Trust and the Baptist Health Foundation of Northeast Florida, both of which are not-for-profit organizations.

Data Availability Statement: The data that support the findings of this study are available on request from the corresponding author. The data are not publicly available due to privacy or ethical restrictions.

\section{References}

1. Adams RJ, McKie VC, Hsu L, et al. Prevention of a first stroke by transfusions in children with sickle cell anemia and abnormal results on transcranial Doppler ultrasonography. $N$ Engl $J$ Med.1998;339(1):5-11.

2. Aguilar-Salinas P, Hayward K, Santos R, et al. Surgical Revascularization for Pediatric Patients with Sickle Cell Disease and Moyamoya Disease in the Prevention of Ischemic Strokes: A Single-Center Case Series and a Systematic Review. World Neurosurg. 2019;123:435-442.e438.

3. Bishop S, Matheus MG, Abboud MR, et al. Effect of chronic transfusion therapy on progression of neurovascular pathology in pediatric patients with sickle cell anemia. Blood Cells Mol Dis.2011;47(2):125128. 
4. Crist WM, Garnsey L, Beltangady MS, et al. Prognosis in children with rhabdomyosarcoma: a report of the intergroup rhabdomyosarcoma studies I and II. Intergroup Rhabdomyosarcoma Committee. $J$ Clin Oncol.1990;8(3):443-452.

5. Dobson SR, Holden KR, Nietert PJ, et al. Moyamoya syndrome in childhood sickle cell disease: a predictive factor for recurrent cerebrovascular events. Blood. 2002;99(9):3144-3150.

6. Fryer RH, Anderson RC, Chiriboga CA, Feldstein NA. Sickle cell anemia with moyamoya disease: outcomes after EDAS procedure. Pediatr Neurol. 2003;29(2):124-130.

7. Griessenauer CJ, Lebensburger JD, Chua MH, et al. Encephaloduroarteriosynangiosis and encephalomyoarteriosynangiosis for treatment of moyamoya syndrome in pediatric patients with sickle cell disease. J Neurosurg Pediatr. 2015;16(1):64-73.

8. Hall EM, Leonard J, Smith JL, et al. Reduction in Overt and Silent Stroke Recurrence Rate Following Cerebral Revascularization Surgery in Children with Sickle Cell Disease and Severe Cerebral Vasculopathy.Pediatr Blood Cancer. 2016;63(8):1431-1437.

9. Hankinson TC, Bohman LE, Heyer G, et al. Surgical treatment of moyamoya syndrome in patients with sickle cell anemia: outcome following encephaloduroarteriosynangiosis. J Neurosurg Pediatr.2008;1(3):211-216.

10. Hasuo K, Mihara F, Matsushima T. MRI and MR angiography in moyamoya disease. J Magn Reson Imaging. 1998;8(4):762-766.

11. Helton KJ, Adams RJ, Kesler KL, et al. Magnetic resonance imaging/angiography and transcranial Doppler velocities in sickle cell anemia: results from the SWiTCH trial. Blood.2014;124(6):891-898.

12. Kennedy BC, McDowell MM, Yang PH, et al. Pial synangiosis for moyamoya syndrome in children with sickle cell anemia: a comprehensive review of reported cases. Neurosurg Focus. 2014;36(1):E12.

13. Lee MT, Piomelli S, Granger S, et al. Stroke Prevention Trial in Sickle Cell Anemia (STOP): extended follow-up and final results.Blood. 2006;108(3):847-852.

14. Ohene-Frempong K, Weiner SJ, Sleeper LA, et al. Cerebrovascular accidents in sickle cell disease: rates and risk factors. Blood. 1998;91(1):288-294.

15. Russell MO, Goldberg HI, Hodson A, et al. Effect of transfusion therapy on arteriographic abnormalities and on recurrence of stroke in sickle cell disease. Blood. 1984;63(1):162-169.

16. Smith ER, McClain CD, Heeney M, Scott RM. Pial synangiosis in patients with moyamoya syndrome and sickle cell anemia: perioperative management and surgical outcome. Neurosurg Focus.2009;26(4):E10.

17. Yang W, Xu R, Porras JL, et al. Effectiveness of surgical revascularization for stroke prevention in pediatric patients with sickle cell disease and moyamoya syndrome. J Neurosurg Pediatr.2017;20(3):232238.

\section{Legends:}

TABLE 1 Patient Characteristics

Legend: $\mathrm{J}=$ Jacksonville $\mid \mathrm{D}=$ Delaware $\mathrm{M}=$ Male $\mid \mathrm{F}=$ Female $\mathrm{Y}=$ Yes $\mid \mathrm{N}=$ No $\mid \mathrm{NA}=$ Not Applicable ${ }^{\mathrm{A}}$ Values based on vasculopathy scores from the SWiTCH grading system ${ }^{11}$ EDAS $=$ Encephaloduroarteriosynangiosis Direct $=$ Direct Surgery $(\text { STA-MCA Bypass })^{\mathrm{B}}$ History of medication ${ }^{\mathrm{C}}$ Time from first transfusion on CTT to last follow-up visit ${ }^{\mathrm{D}}$ Time from first transfusion on CTT to stroke event

FIGURE 1 Flow diagram of patient exclusion and treatment group selection

Legend:

SCD-MMS $=$ Sickle Cell Disease and Moyamoya Syndrome

FIGURE 2 Patient Population Characteristics 
Average Age at First Stroke (2a) Presenting Symptoms (2b) Medications: Aspirin and Iron Chelation (2c) Legend: Abn TCD = Abnormal Transcranial Doppler TIA = Transient Ischemic Attack

FIGURE 3 Surgical treatment group versus conservative treatment group Kaplan-Meier stroke-free survival analysis with Logrank test and follow-up time in years, $\mathrm{p}=0.146$

Legend: Cum Survival $=$ Cumulative Survival Cons $=$ Conservative Treatment Group Surg $=$ Surgical Treatment Group

FIGURE 4 Surgical treatment group versus conservative treatment group Kaplan-Meier stroke-free survival analysis with Logrank test and follow-up time in years, pooled with data from Hall et al ${ }^{8}$ and Yang et al ${ }^{17}$ papers, $\mathrm{p}=0.001$

Legend: Cum Survival $=$ Cumulative Survival Cons $=$ Conservative Treatment Group Surg $=$ Surgical Treatment Group

\section{Hosted file}

Table 1 - Patient Characteristics.docx available at https://authorea.com/users/331752/ articles/458333-surgical-revascularization-in-children-with-sickle-cell-disease-andmoyamoya-syndrome 


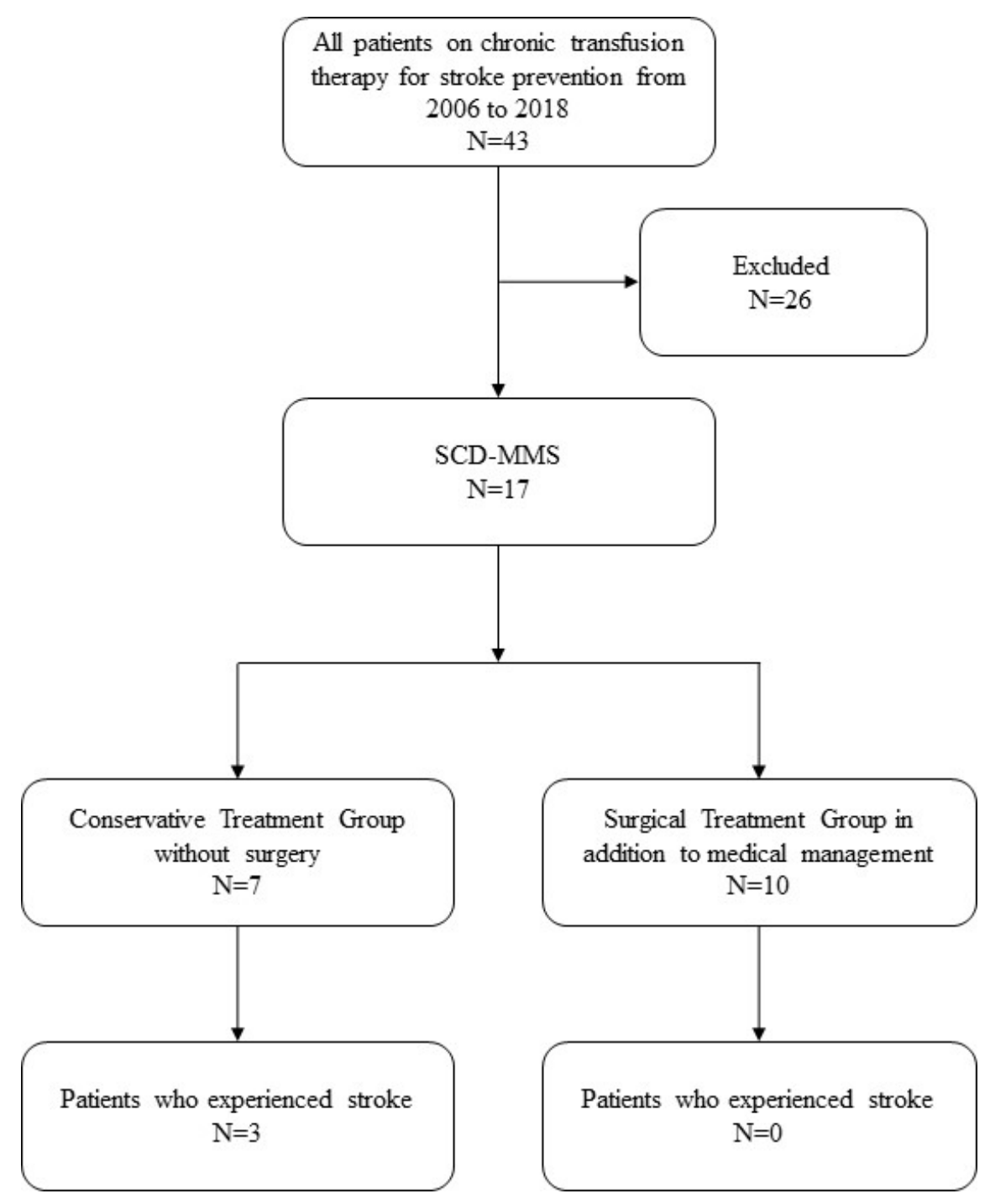



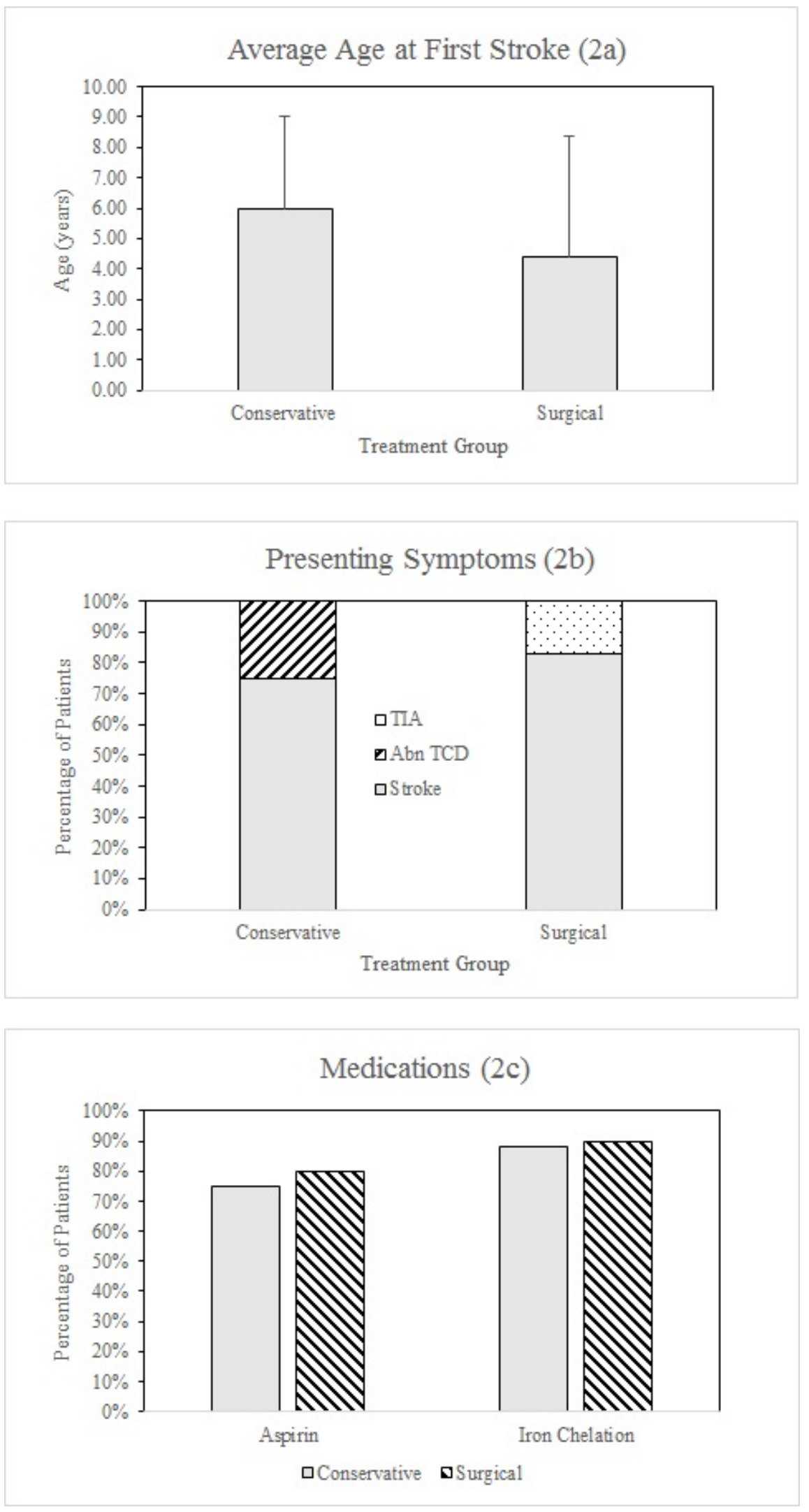

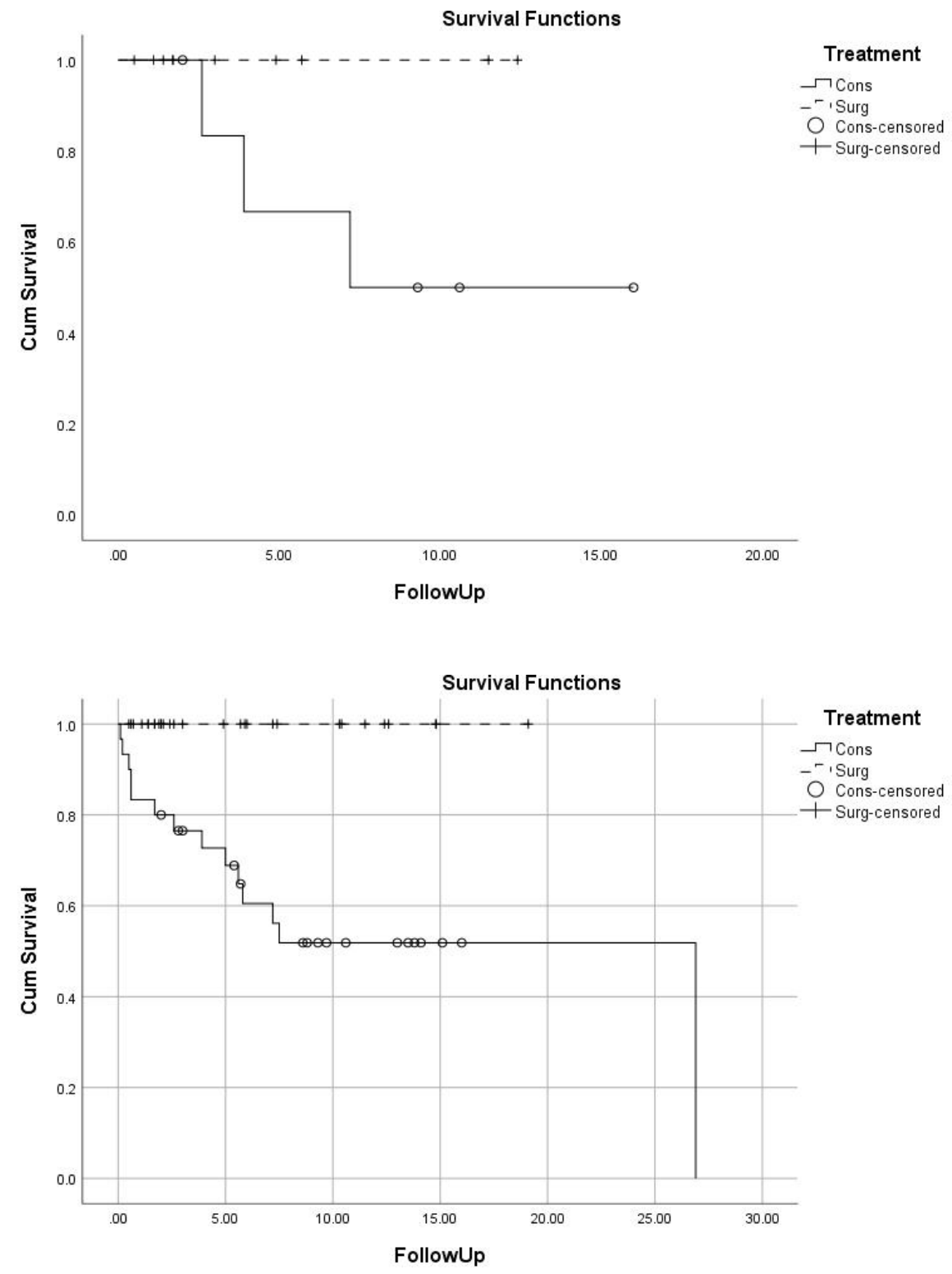